\title{
Document Identifier
}

National Cancer Institute

\section{Source}

National Cancer Institute. Document Identifier. NCI Thesaurus. Code C93399.

The unique identification of a document in a specified context. 